\title{
Optoelectronic NOR gates and rotating drum memory illuminate logic
}

\section{Edward Vogel}

Edward Vogel, "Optoelectronic NOR gates and rotating drum memory illuminate logic," Proc. SPIE 11143, Fifteenth Conference on Education and Training in Optics and Photonics: ETOP 2019, $111432 Z$ (2 July 2019); doi: $10.1117 / 12.2522211$

SPIE Event: Fifteenth Conference on Education and Training in Optics and Photonics: ETOP 2019, 2019, Quebec City, Quebec, Canada 


\title{
Optoelectronic NOR gates and rotating drum memory illuminate logic
}

\author{
Edward Vogel
}

Leonardo's Basement, Minneapolis MN

\begin{abstract}
Optically routed NOR gates using LED and phototransistors have previously been shown by the author to be capable of building all manner of sequential and combinatorial logic gates. Using scissor cut flexible silicone light pipes, logic circuits can be rapidly built and debugged without the use of an oscilloscope or logic probes. An optoelectronic rotating memory has also been developed leveraging a popular "construction toy system" for the structure and mechanism. The capability to build lookup tables and finite state machines greatly expands the possibilities for developing an innovative hands on digital logic education curriculum for middle through high school students.
\end{abstract}

Keywords: Digital Logic Gates, Optoelectronic, NOR Gate Array, Drum Memory, K'NEX

\section{A BLAST FROM THE PAST}

Neon glow lamps and photoconductive cells may be familiar to some of you as an optoisolator circuit, a little archaic perhaps, hearkening back to an era of electrical engineering that preceded silicon and integrated circuits. Nonetheless a circuit that is still in use to this day. ${ }^{1}$

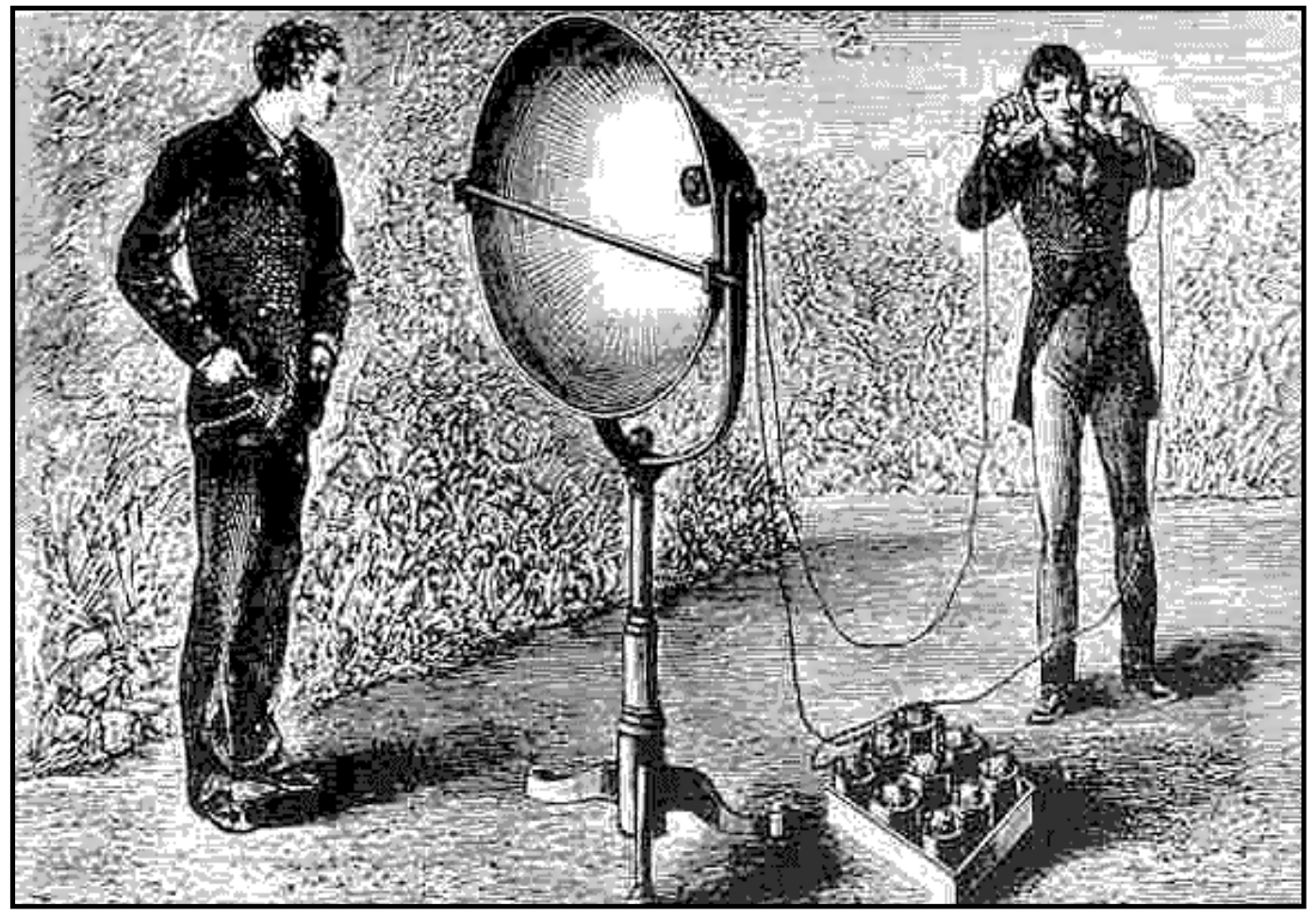

Figure 1 Bell's photophone 


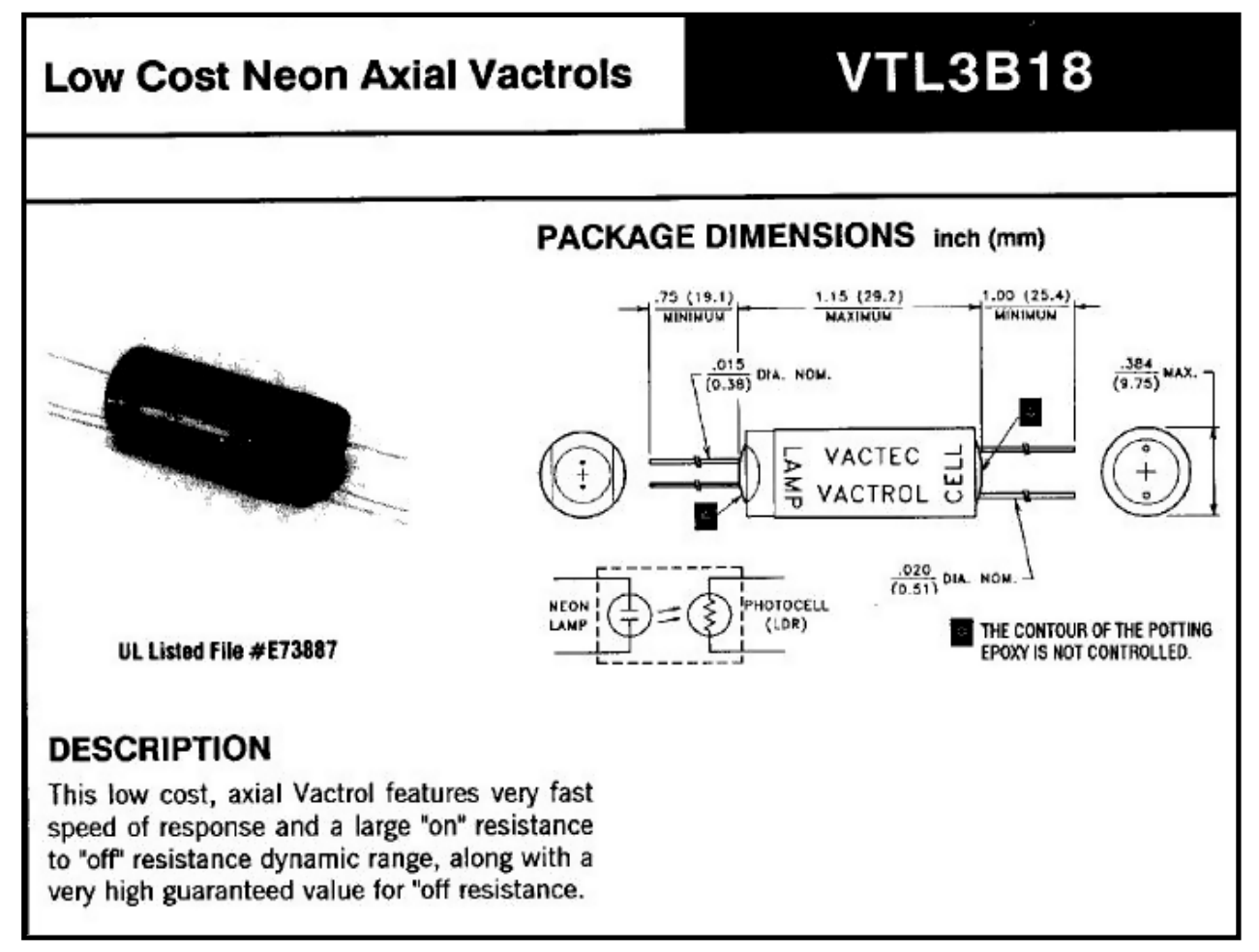

Figure 2 Low cost neon axial vactrol

I would wager few of you know of the circuit in Fig. 2 as the basis of a family of digital logic capable of implementing combinatorial and sequential logic circuits. Wanna bet? I did.

I discovered these circuits as part of a wager regarding the capabilities of electronic technology sans silicon (or other highly purified, can't easily make in the garage or basement) semiconductors. The "discussion" began with me griping about not being able to use a special function IC in an instrument to detect bearing failure in Navy nuclear reactor feed pumps. It was not second sourced and could not be used, back to the drawing board. I conjectured that our being reliant on someone else to make our devices was a weakness and we should look for a technology that would allow us to fabricate our own. The response from my coworkers was "if there was anything else, someone would have discovered it already, get back to work." I persisted, looking into ionic crystal amplifiers using halide salts which were researched at the turn of the century. How fun is that? You could look inside the crystal and see the color centers move in response to electrical stimulation. One of my coworkers agreed to a wager; \$1.00 US that I could "NOT" find a reliable solid state switching or amplifier device using technology existing prior to 1900.

Game on!

Ionic crystals were out from the get go because they are not reliable and required being heated with a Bunsen burner. I decided to start experimenting with photoconductive cells. Photoconductive selenium was widely know by 1880 . Bell's photophone shown in Fig. 1 was not in wide use but what cool picture.

Maybe if I used one photocell to control the brightness of a lamp and then couple that lamp to several cells I could get a significant current gain. I got one experiment with a current gain of four, I think. I wasn't really keeping a notebook. What if I made an inverting amplifier? Maybe that would help. I went to Radio Shack to get more lamps and photoconductors, "Hey look neon bulbs at 110 VAC how could that not be fun to play with."

I put together the circuit shown in Fig. 3 because it looked like something to do with almost no parts to be the input stage of the inverting amplifier. I discovered that neon lamps are not linear devices. I did not know this at the time. It's a switch, 
OK. "This is kind of interesting." I built two more and arranged them in a sort of a triangle. The lamp of one circuit closely situated to the photocell of the next. The lights were doing something obviously, they were not as bright as normal. Was it a ring oscillator? I wasn't sure because it looked more like a flicker than a blink so I built four more. With seven circuits it was pretty evident they were blinking. Hook up an oscilloscope to the electrical side of the circuit? Perish the thought. Edison and Bell didn't have one and I wasn't going to use one either!

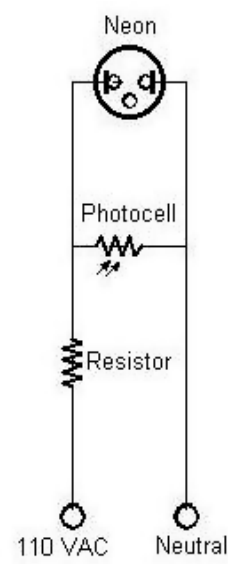

Figure 3 Neon Lamp and Photoconductor NOR Gate

\section{MUST YOU ALWAYS BE SO NEGATIVE? YES? THANKS!}

My coworkers were pretty negative about the idea of anything else when it came to device technology. How appropriate that I should rediscover a NOR gate. "No" means much more than "no" it seems. In propositional logic it is a fundamental property preceding OR, AND, XOR and the rest of the logic functions. All of them can be made from NOR. Also it doesn't matter how many AND, OR and XOR gates you chain together, it ain't gonna oscillate. No way. No How. Nyet. Nada. No.

Getting back to the past. The circuit Fig. 3 was relatively well known by the late 1950s and is described in the venerable “GE Glow Lamp Manual” shown in Fig. 4.

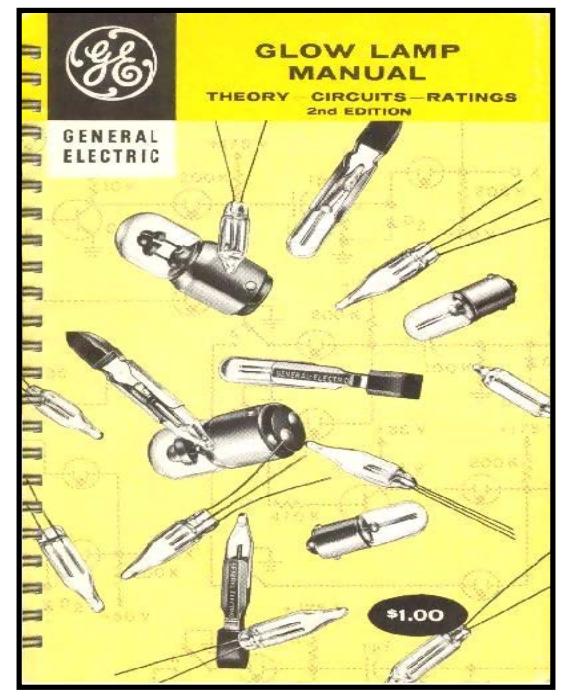

Figure 4 "GE Glow Lamp Manual” 
It is well worth Googling. A whole chapter on neon lamps and photoconductor circuits. I also found articles in Electronics Magazine by (Radio Corporation of America) RCA and IBM researchers.

Here is a classic:

\section{J. L. Patterson, “Will Neon Photoconductors Replace Relays in Low-Speed Logic?”2}

Sometimes getting an article as hard copy can be a little tricky. Google books result for "Find in a library (near you)": University of Zululand KwaDlangezwa, 3886 South Africa -9200 miles

Turns out there is a copy at University of Minnesota. I checked it out through Hennepin County Interlibrary loan. I must pause here to give Hennepin County Library some kudos here. They provide access to many of the databases that would typically require the use of a college library and the attendant tuition and fees.

An interesting read to say the least. Will neon photoconductors (Ne-PC) replace relays in low speed logic? They haven't yet as far as I am able to tell:

Ne-PC had their niche' in the music industry but not replacing relays. Perhaps Ne-PCs did replace relays somewhere once upon a time gone by. I believe there were punch card readers using neon lamps and photocells. Doing some decode logic might have made sense. If you do check out the book I have cited you will also see (Electroluminescent-Photoconductor) EL-PC circuits, that is electroluminescent photoconductor circuits. Interesting circuits, however all of them routed the light by arranging the Ne-PCs or EL-PCs on printed circuit boards so that Ne-PC1's output aligned to Ne-PC2's input to form logic gates. I didn't like this, I wanted to design one circuit, build a bunch and then make them sit up and bark. I wrestled with this in my mind for a week or two. The answer came to me as I sat down in an airline seat that was about to whisk me away from my Ne-PCs for a week in glorious Sunnyvale to babysit cell phone base stations under test.

Why not route the light between circuits with fiber optics or some other Total Internal Reflection (TIR) method?

Because I am on this airplane for one thing. One must pay the bills of course. When I got home that Friday night I purchased, cut, bent and polished some 1/8” acrylic square bar stock. I scotch taped them to three Ne-PC circuits and sure enough it oscillated. OK, I have a clock circuit and some confidence what is next?

Combinatorial and sequential logic of course.

\section{LIGHTS, CAMERA, ACTION!}

Let's have a look at some logic gates. Electrically these are simple circuits and easy to draw in most cad packages, I used LTSpice. Routing the light in a CAD package has eluded me so what I am showing here is a little bit primitive; cut, copy, paste using MS Paint. After each circuit diagram there is a link to a YouTube video showing the circuit in action, to best of my abilities and my Vivitar DVR 510N. Apparently painting the thirty six NOR gate board black makes the camera think it is a low light condition which makes for jumping back and forth between color video footage and "night vision" which is black and white. I also have no tripod. The videos are pretty fun nonetheless, so if my writing is getting boring skip ahead and click on the YouTube links.

Rules of the Route:

- Thick yellow lines originate at an energized neon lamp and terminate at a photocell.

- Thick black lines originate at an unenergized neon lamp and terminate at a photocell.

- Photocells can accept up to three inputs.

- A yellow line at a photocell means the photocell is being illuminated and is conducting which extinguishes the neon lamp it is electrically connected to.

- A black line at a photocell means the photocell is not being illuminated and is not conducting and the neon lamp it is electrically connected to is lit up.

- Yellow trumps black. It does not matter how much dark you route, light wins out. At least in this instance. 
These circuits in principle are "on at dusk, off at dawn" night lights? The ones you see in the store are typically a photoconductive cell controlling a triac supplying current to an incandescent bulb. If you don't feel like building this circuit several times (seven-ish if you want to make a really cool clocked D Flip Flop) you can go to the store and buy a bunch of night lights.

Principle of Operation circuit figure and description:

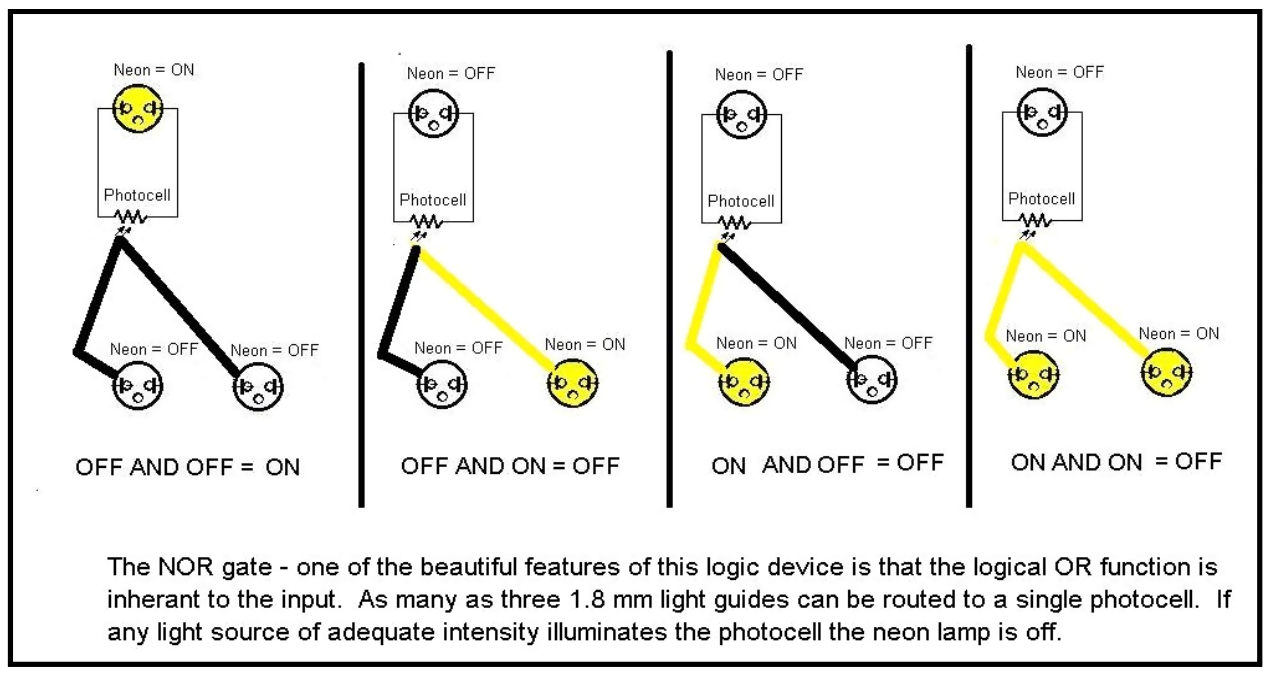

Figure 5: NOR Gate figure and description ${ }^{3}$

I don't have video for this one. Instead here are my "Christmas Lights” a ring oscillator of thirty five circuits: ${ }^{4}$

OR Gate figure and description:

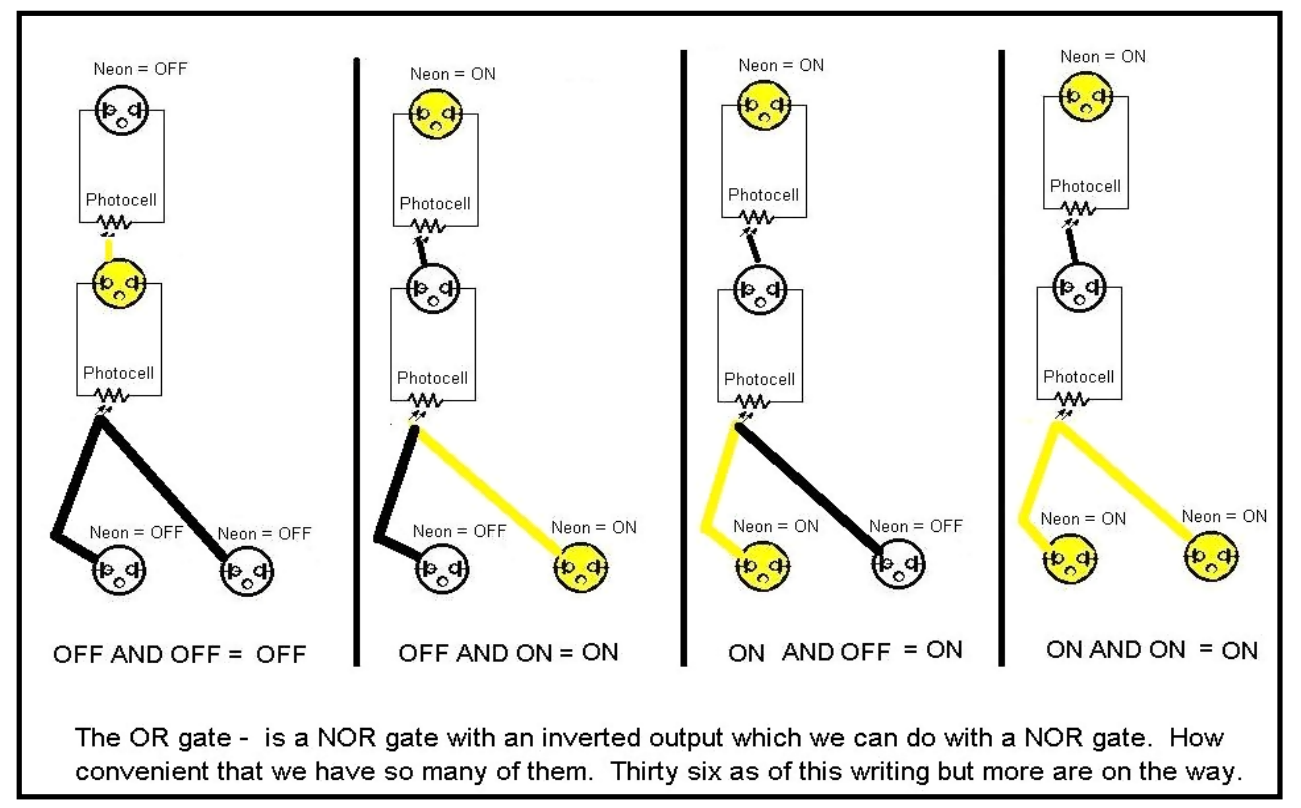

Figure 6: OR Gate figure and description

OR in the routing video ${ }^{5}$. 
AND Gate figure and description:

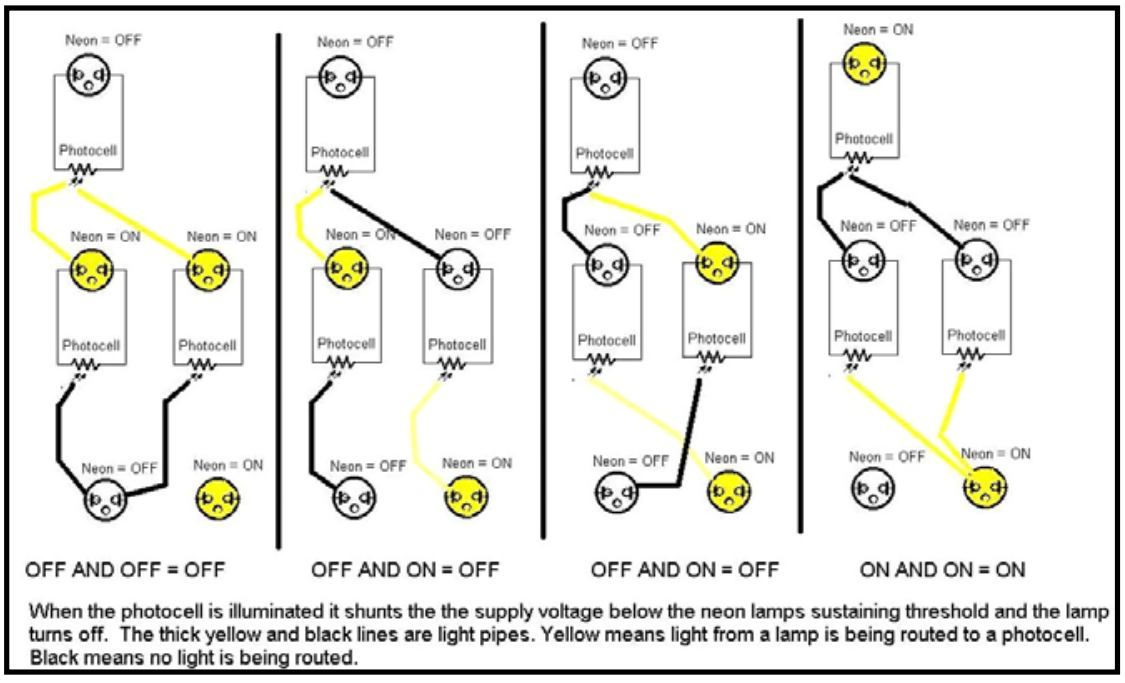

Figure 7 AND Gate figure and description

AND Gate in Action video. ${ }^{6}$

The first job of the engineer is to show the manager that the princely sums being poured into a project show a tangible result. Blinking a lamp is the standard of showing that the money spent is actually doing something. Imagine though if the very circuit you are basing your designs on is in itself a blinky light. One of these circuits fed back on itself does oscillate but it isn't easy to see. It also is not easy to draw in MS Paint so I am using screen shots of a Cedar Logic simulation to show the propagation. ${ }^{7}$ Three gates gets close to being something you can see as a "blink." More is better though and if you do some creative routing you can see some lovely patterns.

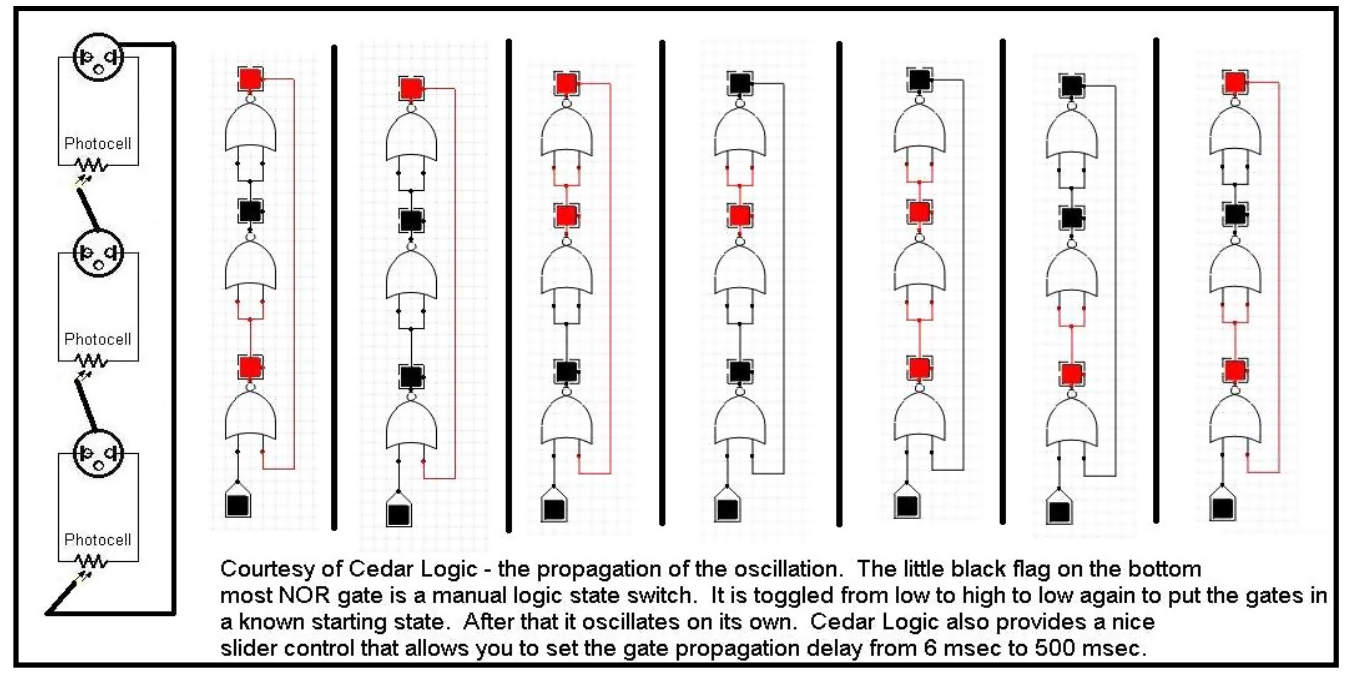

Figure 8 Oscillator

Here is an earlier oscillator. It also is a good view of the construction of the device should you choose to build one. "No" is a very useful concept. Who wants a gaggle of preening "yes men" fawning over him or her all of the time? Agreeing with everything (conditionally at any rate). Being wrong (goodness knows I am good at that) is a pretty negative experience but it also contains much more information than being right or perhaps better stated "not being wrong." If I ever pursue a doctorate I think it will be in "Being Wrong." 


\section{SEQUENTIAL LOGIC}

Combinatorial logic is great of course and I could go on to show how this particular instance of a NOR gate can form XOR and XNOR gates and half adders, full adders, black adders and majority detectors. If you have NOR gates, you have all of the combinatorial logic functions. Sequential logic is another kettle of fish. Its all in the timing.

I must take a moment here to plug Cedar Logic and Source Forge. I do volunteer work and I like what I am teaching/facilitating to be inexpensive and easy to get. The internet has certainly made that easier and Source Forge is a great resource for real tools, real cheap and real quick. Cedar Logic is a good example of this. I have looked at inexpensive logic simulator "off and on" over the years but they all seemed to have a problem with ring oscillators and cross coupled NOR gates to form latches. Cedar Logic not only works for NOR latches its authors made themselves available to explain how to make the oscillator work. These guys are putting in the extra effort and deserve some kudos.

Making a proper clocked D Flip Flop is pretty much where I left my work on these lovely blinkies back in 1997 . The texts I was reading did not address using basic gates to build anything more complicated than an RS Latch and a D Latch. The implication was that somehow the clock had to be introduced into the circuit as an edge generated from a differentiated signal of the system clock. Not easily done with light. Yes, I could have AC coupled the photocell to the neon lamp for a few special NOR gates situated strategically throughout the board and ran the whole thing on DC but that just seems like, no way, uh-uh. No specials, blech. It needs to be simple.

AC coupling is the way to go if you are working with transistors and you want to use as few as possible. However it is not the only way to get where you want to go. Particularly if you want to go to the moon. The Apollo Guidance Computer $(\mathrm{AGC})^{8}$ was made primarily from NOR gates to simplify the task of qualifying circuits for use in the extreme environmental conditions of spaceflight. ${ }^{9}$ One Integrated Circuit (IC) for hundreds of logic gates and one qualification task. Simple!

The folks at The Virtual AGC were of great help.

The Apollo Guidance Computer for me was "if NASA and MIT can build a powerful computer largely from NOR gates, there must be something I am missing." I looked at some schematics of the AGC and didn't really see a clocked D Flip Flop. It appeared that they may have been pioneering asynchronous CPUs. Or likely I am just a lazy schematic reader when I don't have hardware in front of me. I fooled around with Cedar Logic elaborations of NOR latch master and slave arrangements, two phase clocks and "pulse extraction" of the system clock.

D Flip-Flop Using NOR Latches:

http://www.play-hookey.com/digital/alt_flip_flops/d_nor_flip-flop.html

I routed one up and it worked. ${ }^{10}$ See Figures 9 and 10. 


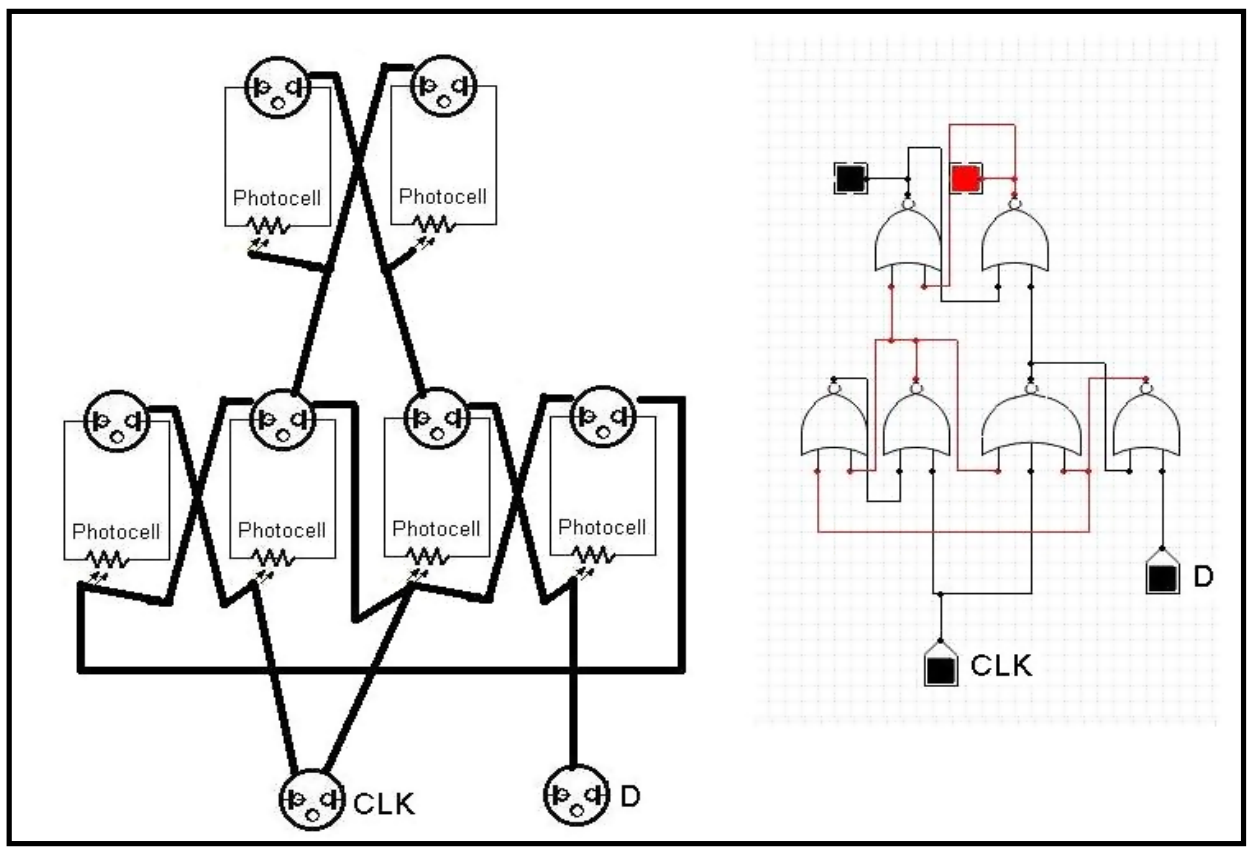

Figure 9: Clocked D Flip Flop

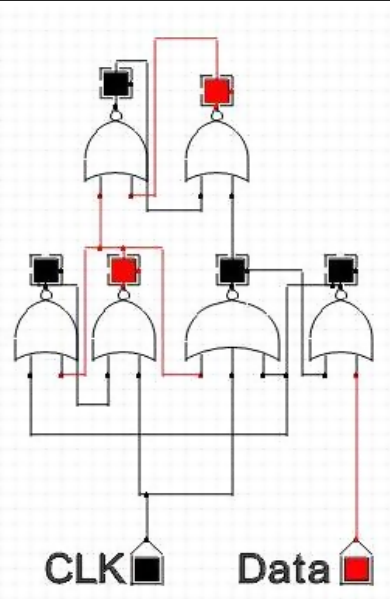

Previous State

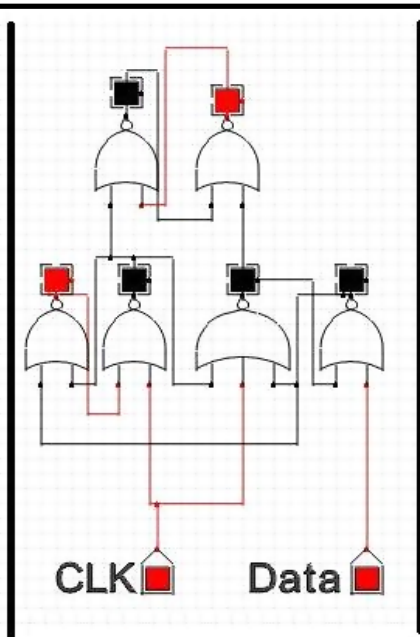

Clock Goes ON

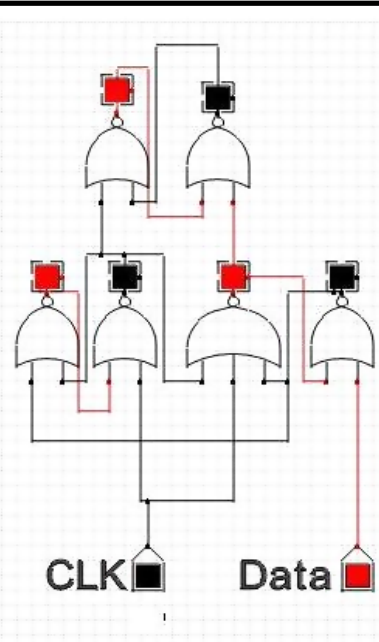

Clock Goes OFF

I am not as good at drawing as the folks at Cedar Logic: http://sourceforge.net/projects/cedarlogic/ They make a nice little simulator for free.

Figure 10: Clocked D Flip Flop States

\section{LEONARDO'S BASEMENT - BE SAFE, BE NICE AND HAVE FUN. ALSO THINK BIG.}

Things happen in life that distract from completing tasks sometimes for good sometimes for ill. After bouncing around several jobs and locales for ten years and change I landed in Minneapolis and met up with the hands on folks at Leonardo's Basement. ${ }^{11}$ 
Where we make big ideas happen. For instance "proving" that "math is fun" at the Minnesota State Fair using a giant version of the SOMA Cube puzzle. This project found its way into an illustration about self organizing mechanisms in biology in Nature Chemical Biology "Cooperativity in macromolecular assembly"12 by James R Williamson of Scripps Research Institute as shown in Figure 11.

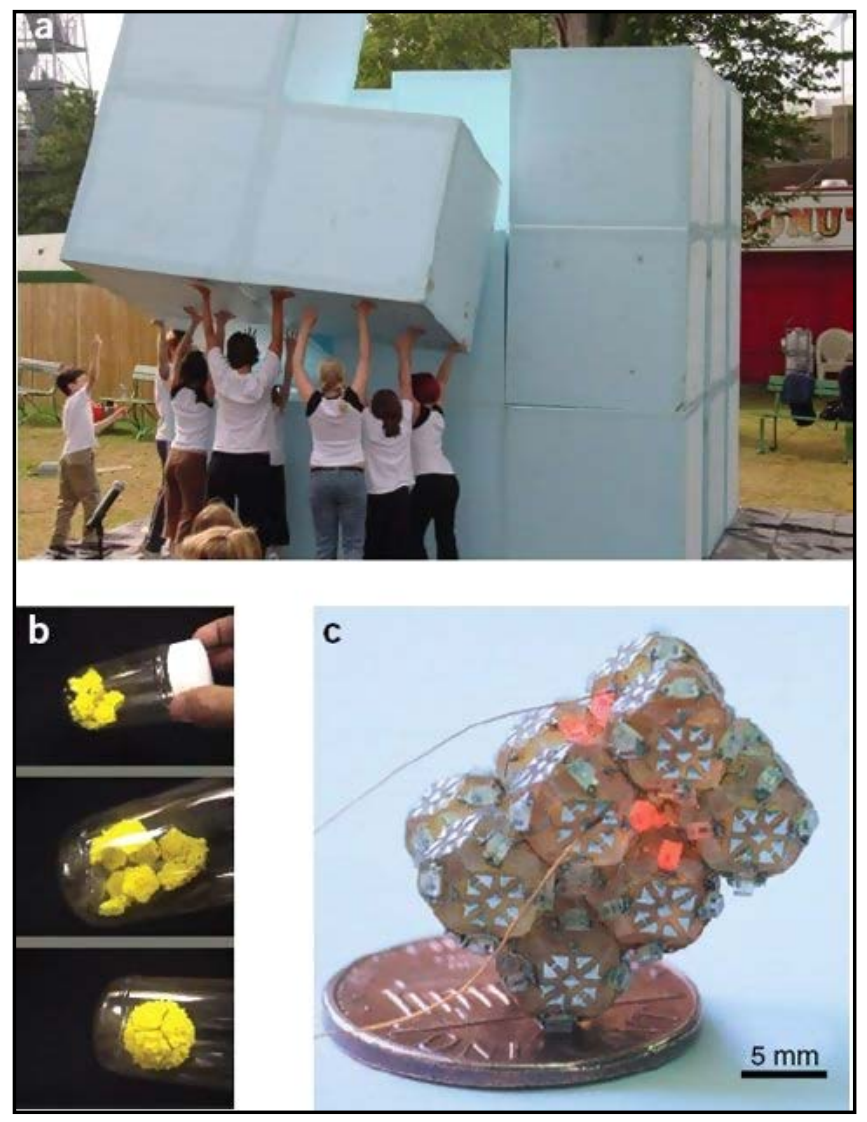

Figure 11 Giant SOMA Puzzle as self assembly analogy

I tinkered around with getting some optoelectronic digital circuit workshops into Leonardo's summer sessions but it was hard to get time off from work and more importantly to me I wanted to build circuits that could do something more than demonstrate principles. Something like a simple vending machine for instance. One of the instructors did work with one or two students and volunteers to build and install "HAL" in our TARDIS at the shop. This picture is of the TARDIS transported to an out door event.

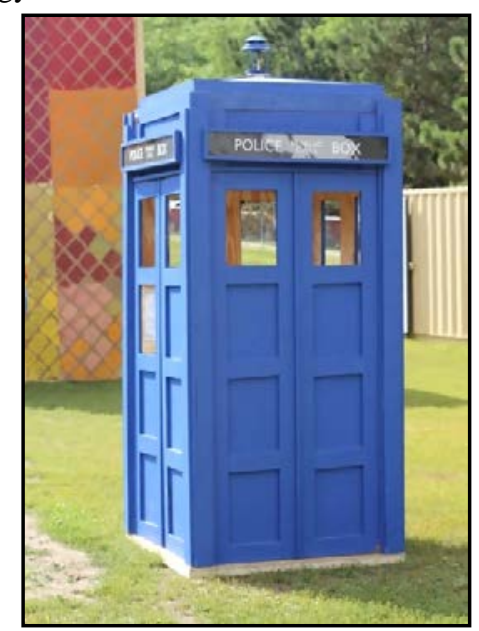




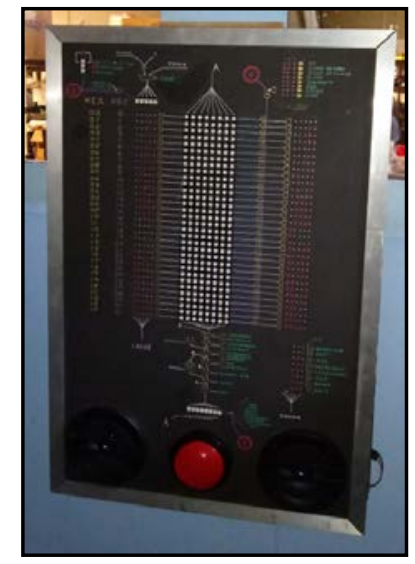

Figure 13 HAL Panel 3

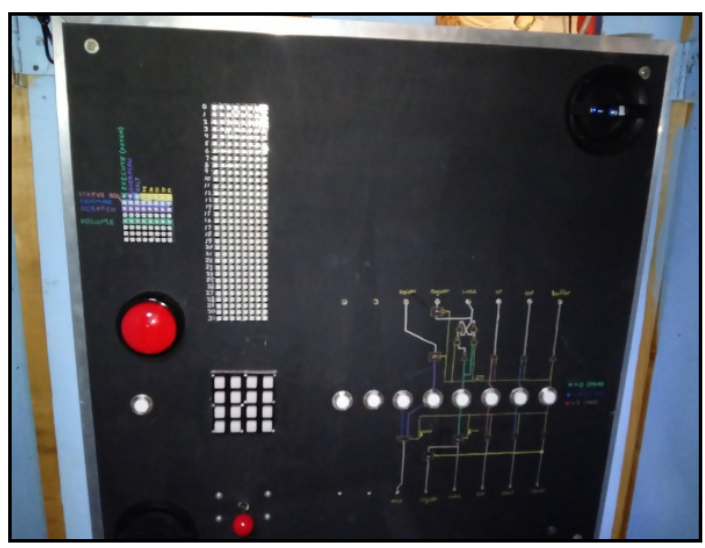

Figure 14 HAL Panel 4

The HAL idea is to "spell it all out" through bites size interactive "panels".

Panel 1 - hands-on "water analogy" of electricity. With pressure (voltage), current flow and obstructions in the pipes (resistors). Kids could play with this to see how "electricity" works.

Panel 2 - with valves (transistors) then logic gates (nands, inverters, nors). Again, hands-on.

Panel 3 - is more logic gates built up with these nand/nor's etc. (Like an adder adder, binary to decimal encoders, etc.)

Panel 4,5 and 6 - HAL doing something meaningful, like controlling a simple xy table or something.

\section{DRUM ROLL PLEASE!}

\subsection{Rotating Drum}

One of my first thoughts was to make Look Up Tables (LUTs). LUTs are a great way to create lots of combinatorials circuits of various widths and complexity. Pretty much the basis of Xilinx (Field Programmable Gate Array) FPGA. Unfortunately storing each bit requires a latch and associated combinatorial gates to make it read/writeable at a unique address. Back to the drawing board or in my case back to the library. Surely there were clever tricks for storing data before transistors made memory to chep to meter. 


\subsection{Drum concept discovered at UMN History of Computing Library}

The University of Minnesota has the Charles Babbage Institute a history of computing library so I took an afternoon off from my medical device job and paid them a visit. ${ }^{13}$ There I found the key to working with limited gates, the rotating drum memory. From Babbage to Atanasoff to finally interestingly my dad. He didn't invent any computer hardware but he did begin his computer software career on the IBM650. It took me a while to grasp where the logic gate saving came in though reading these papers. Finally it dawned on me that you didn't control the drum's position to find the data at a particular address. You just let it spin and wait for the address on you put into the latches to match the address on the drum and then read out the data to a register. ${ }^{14,15}$ But how to build the address comparator? Particularly out of NOR gates. After some Googling around I discovered that a 2 input comparator can be made from XNOR gates which used four NOR gates each. This seems reasonable now how to test it.

\subsection{Simulation with CircuitVerse}

About the same time I discovered a free online circuit simulation site. ${ }^{16}$ This is important to me as again I work with kids with limited resources so free and online is critical to getting them interested and able to work on their own. At the time it did not support feedback so this first go mainly tests the principle of operation but it works:

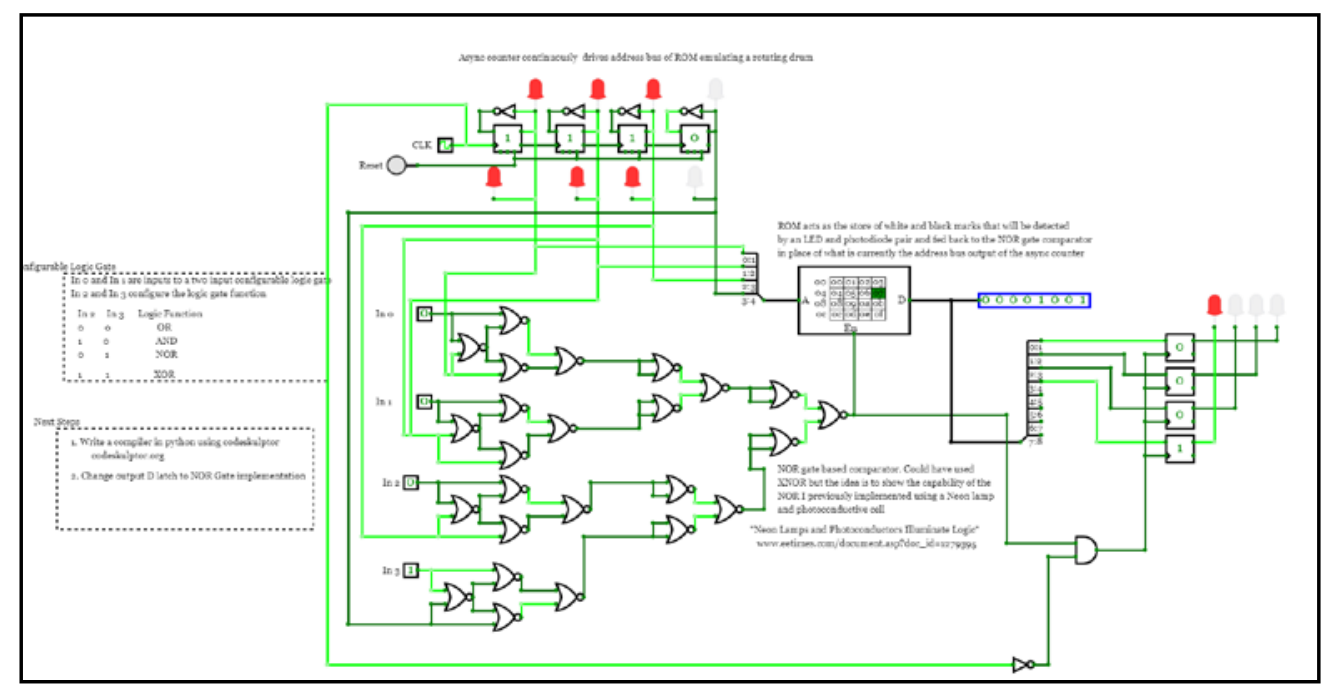

Figure 15 Rotating Drum Memory Simulation

\section{DISCOVERING K'NEX AND FIRST DRUM CONSTRUCTION}

It works in principle but what about in the real world? I built the first hardware with very primitive spin by hand drums made from Pringles cans and barbeque skewers. ${ }^{17}$ Also all NOR gates including the D Latches.

Computer on a Chip (can):

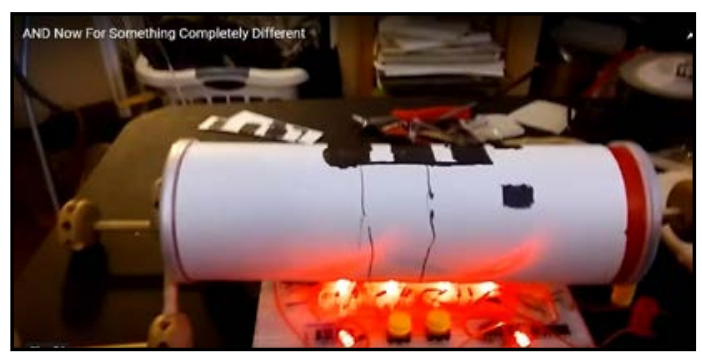

Figure 16 Computer on a Chip (can) 
So it works but it wasn't anything reliable that I would share in a digital logic workshop. Then I remembered seeing a K'Nex construction kit for a Ferris wheel with an electric motor. Seemed worth a try. This is starting to look like something that makes sense. ${ }^{18}$

Drum LUT 2.0:

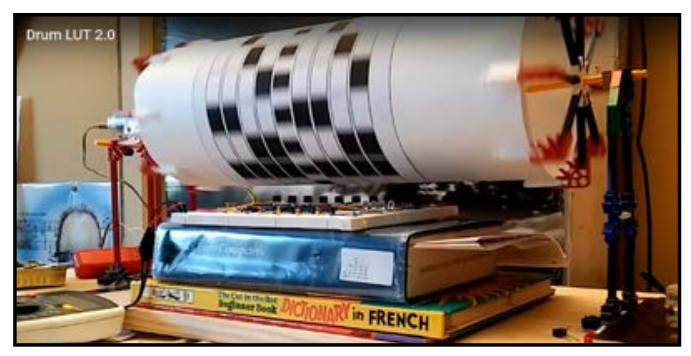

Figure 17 Drum LUT 2.0

\section{CONCLUSION - IDEAS FOR A HANDS ON COMPUTER SCIENCE ACTIVITY}

- Optoelectronic Combinatorial Gates

- Optoelectronic Sequential Gates

- LSI Combinatorial and Sequential Gates

- Rotating Drum Memory 1 - the basics of the HW and how get the data on the drum

- Rotating Drum Memory 2 - Write a state machine compiler using codesculptor ambitious but worth it

- Rotating Drum Memory 3 - RAM

\section{REFERENCES}

[1] Vogel, E., "Neon lamps and photoconductors illuminate logic,“ Electronic Engineering Times, (2012) https://www.eetimes.com/document.asp?doc_id=1279395\#

[2] J. L. Patterson, “Will Neon Photoconductors Replace Relays in Low-Speed Logic?,” Electronics, 36:18, p 46-49 (1965).

[3] Vogel, E., “NOR Gates”, http://youtu.be/n1Yoi3GIyW4

[4] Vogel, E., “ChristmasLights”, http://youtu.be/m6MZZ45RIqI

[5] Vogel, E., “OR In The Routing”, http://youtu.be/XVu-MIdnUdo

[6] Vogel, E., “AND Gate”, http://youtu.be/EmSeBPJLD_0

[7] CEDAR Logic Simulator, https://sourceforge.net/projects/cedarlogic/

[8] The Apollo Guidance Computer, http://en.wikipedia.org/wiki/Apollo_Guidance_Computer

[9] Virtual AGC, http://www.ibiblio.org/apollo/

[10] Vogel, E., “D Flip Flop Q NOT to D” http://youtu.be/RkpPbfy900M

[11] Leonardo’s Basement, https://leonardosbasement.org/

[12] Williamson, J. R., “Cooperativity in macromolecular assembly,” Nature chemical biology, 4(8), 458 (2008).

[13] Charles Babbage Institute - http://www.cbi.umn.edu/ 
[14] Carlson, W. B., Lyle R. Johnson, John H. Palmer, and Emerson W. Pugh [IBM's Early Computers], Cambridge, Mass.: MIT Press, (1985).

[15] Ceruzzi, P. E., “Jacquard's Web: How a Hand-Loom Led to the Birth of the Information Age,” Technology and Culture, 47(1), 197-198 (2006).

[16] CircuitVerse_ Online Digital Logic Circuit Simulator, https://circuitverse.org/

[17] Vogel, E.,’AND Now For Something Completely Different” https://youtu.be/XV3E-NSQuUE

[18] Vogel, E., “Drum LUT 2.0” https://youtu.be/KoeOXJnhEE8 